\title{
On morphological operators based on rank filters
}

\author{
Pierre Soille*
}

EC Joint Research Center, Space Applications Institute, TP 262, I-21020 Ispra, Italy

Received 10 August 1999; received in revised form 10 August 2000; accepted 2 January 2001

\begin{abstract}
Rank order morphological operators have been designed to better cope with shape variations and noise than the corresponding operators with plain structuring elements. In this paper, starting from a brief historical overview of rank order filters and erosions/dilations in digital image processing, rank order based morphological operators are surveyed. We also highlight the relations between these operators and show that many are similar if not equivalent. An extensive bibliography is provided. (C) 2001 Pattern Recognition Society. Published by Elsevier Science Ltd. All rights reserved.
\end{abstract}

Keywords: Mathematical morphology; Rank filters; Order statistics; Median; Opening; Closing; Hit-or-miss; Image filtering; Minkowski

\section{Introduction}

Medians and other order statistics have since long been used and investigated in statistics as alternatives to sample means in estimation of population means $[1,2]$. Moving median filtering has been suggested by Tukey $[3,4]$ (Limited Preliminary Edition 1971 referred to in Refs. $[8,12,29]$.) as a filtering tool in economic time series analysis by letting a window move over points of the series and replacing the value at the window centre with the median of the original values of the points falling within the window. First applications of running median filters to digital ${ }^{1}$ image data have been described in 1974 for correcting scanner noise by removing salt and pepper artifacts [8] and in 1976 for enhancing edge gradients by removing spurious oscillations [9]. In signal processing, median filtering has been first applied to digital speech signals for eliminating pitches [10] and transmission errors [11].

Order statistic filtering is performed similar to median filtering: a window is moved over the picture and the $k$ th order statistic of the values within the window is com-

\footnotetext{
* Tel.: + 39-0332-785-068; fax: + 39-0332-789-803

E-mail address: pierre.soille@jrc.it (P. Soille).

URL: http://ams.egeo.sai.jrc.it/soille.

${ }^{1}$ For analog median and rank filters, see Refs. [5-7].
}

puted. In image processing, order statistic filters are also known as percentile filters [12, p. 195, 13] and, more commonly, as rank filters [14] or rank(ed) order filters ${ }^{2}$ [17-19]. One of the earliest published application of rank order filters in image processing is due to Heygster [20] in 1978 and deals with the filtering of cell pictures. Many additional references on median and order statistic filters can be found in the following three review papers [21-23]. Hardware architectures for rank order filters are proposed in [24-27]. A fast algorithm for rank filters based on the moving histogram technique [28,29] initially introduced for median filters is detailed in Ref. [30] and adapted to arbitrary shaped structuring elements in Ref. [31].

In discrete mathematical morphology [32-36], the minimum and maximum ranks play a key role since they correspond to the fundamental erosion and dilation operators (also called $\min$ and $\max$ operators in Refs. [37,38] as a generalization of the binary shrink and expand operators). The purpose of this paper is to provide a detailed review of all morphological operators based on rank order filters. We also highlight various

\footnotetext{
${ }^{2}$ Note that the term order filters [15] concerns a generalization of rank order filters whereby the sorted array of signal values is mapped by a real-valued not-necessarily linear function. When the function is linear, order filters correspond to the $L$-filters proposed earlier [16].
} 
links between these operators. Alternate morphological approaches aiming at dealing with more flexible structuring elements (e.g., fuzzy mathematical morphology, see Ref. [39] for a survey) are out of the scope of this paper.

The paper is organised as follows. Definitions and notations of rank and erosion/dilation operators are presented Section 2. Their relations together with other related operators are then discussed. Section 3 reviews opening/closing based on rank filters and analyzes their relations. Section 4 details rank based hit-or-miss transforms and shows that they can all be viewed as instances of the vector rank order operator. A summary table is presented in the conclusion.

\section{Basic definitions, notations, and links}

\subsection{Rank filters}

The output value of a rank filter of rank $k$ with a finite discrete window of arbitrary shape $B$ is obtained by sorting in ascending order the pixel values falling within the window centred at the considered pixel and selecting the $k$ th value in the sorted array. We denote by $\zeta_{B, k}$ the rank filter of rank $k$ and window (structuring element) $B$, i.e., $k \in\{1,2, \ldots, n\}$ where $n=\operatorname{card}(B)$ is the number of elements (cardinal number) of $B$.

Rank filters are nonlinear, increasing, ${ }^{3}$ and translation invariant operations. They also satisfy the threshold decomposition property [40] and therefore commute with monotonically increasing functions (anamorphoses) [41]. By construction, the following ordering relation is always satisfied: $\zeta_{B, 1} \leqslant \zeta_{B, 2} \leqslant \cdots \leqslant \zeta_{B, n}$. Each rank filter $\zeta_{B, k}$ has a corresponding dual $\zeta_{B, n-k+1}$ with respect to complementation C, i.e., $\zeta_{B, k}=\mathrm{C} \zeta_{B, n-k+1}$ C. The median filter requires $n$ to be odd. It is then defined as the rank filter for the rank $k=(n+1) / 2$. The median filter is the only self-dual rank filter.

\subsection{Erosion and dilation}

As already pointed out in Ref. [42], considerable confusion has arisen regarding the definitions and notations of the erosion, dilation, and Minkowski subtraction. This confusion has two reasons. First, while there is a unique definition for the erosion, there exist two different definitions for the dilation and both are widely used in the literature. Second, identical symbols are used by different authors to mean different things. All these issues are detailed hereafter.

\footnotetext{
${ }^{3}$ The increasing property is sometimes called the stacking property, see for example Ref. [40].
}

Everyone agrees on a common definition for the erosion, i.e., the erosion of a set $X$ by a structuring element $B$ is the locus of the points $x$ such that when $B$ is centred at $x$ it is included in $X$ (or, equivalently, as the intersection of the translations $X_{-b}$ of $X$ by the opposite vectors $-b$ of $B$ ):

erosion of $X$ by $B=\left\{x \mid B_{x} \subseteq X\right\}=\bigcap_{b \in B} X_{-b}$.

There exist however two distinct definitions for the dilation. In one of the earliest publication [43, pp. 16,17] devoted to mathematical morphology, the dilation is referred to as Serra's transform by dilation (and the erosion as Serra's transform by erosion). This dilation is defined [33, p. 43] as the locus of the points $x$ such that when $B$ is centred at $x$ it has a non-empty intersection with $X$ :

dilation of $X$ by $B$ (first definition)

$$
=\left\{x \mid B_{x} \cap X \neq \emptyset\right\}=\bigcup_{b \in B} X_{-b} .
$$

The second definition is very similar but not identical to the first because it is defined as the locus of the points $x$ such that when the reflection of $B$, denoted by $\breve{B}$, is centred at $x$ it has a non-empty intersection with $X$ :

dilation of $X$ by $B$ (second definition)

$$
=\left\{x \mid \check{B}_{x} \cap X \neq \emptyset\right\}=\bigcup_{b \in B} X_{b} .
$$

This latter definition originally appeared in Ref. [44] and has been adopted by numerous researchers following Ref. [45]. Notice that a similar type of confusion occurs in linear signal processing where a convolution by a given kernel is often defined as the weighted sum of the image values falling within the kernel itself without reflecting it beforehand. If the reflection is neglected, this 'convolution' is in fact a cross-correlation with the kernel, or, equivalently, a convolution with the reflected kernel $[46$, p. 86]. Indeed, it is a common practice to refer to the impulse response of a filter as the filter itself.

In morphology, even more confusion arises when one comes to the notations because identical symbols have been used for different operations. Indeed, the commonly agreed definition for the erosion coincides with the Minkowski subtraction originally defined by Hadwiger [47, p. 212]. The Minkowski subtraction is usually denoted by - or $\Theta$, in accordance with the signs + or $\oplus$ used for the Minkowski dilation defined long before Ref. [48]. However, in Ref. [43] and the subsequent reference publications [32,33], the erosion of $X$ by $B$ is denoted by $X \ominus \check{B}$, i.e., the Minkowski subtraction is redefined with the reflected set! A functional notation of erosions and dilations (resp.) has more recently been adopted (e.g., Refs. [35,36]). In this case, the notation for 
Table 1

Basic transformations of a set $X$ by a structuring element $B$

\begin{tabular}{llc}
\hline Transformation & Definition & Notations \\
\hline Minkowski addition [48] & $\cup_{b \in B} X_{b}$ & $X+B$ or $X \oplus B$ \\
Minkowski subtraction [47] & $\bigcap_{b \in B} X_{-b} \bigcap_{b \in B} X_{b}$ & $X-B$ or $X \ominus B$ \\
Redefined Minkowski subtraction [33,43] & $\bigcap_{b \in B} X_{-b}$ & $X \ominus B$ \\
Erosion (1st notations, e.g. [33,43]) & idem & $X \ominus \bar{B}$ or $\varepsilon_{B}(X)$ \\
Erosion (2nd notations, e.g. [44,45]) & $\cup_{b \in B} X-b$ & $X \ominus B$ or $\varepsilon_{B}(X)$ \\
Dilation (1st definition, e.g. [43,33]) & $\cup_{b \in B} X_{b}$ & $X \oplus \check{B}$ or $\delta_{B}(X)$ \\
Dilation (2nd definition, e.g. [44,45]) & & $X \oplus B$ or $\delta_{B}(X)$ \\
\hline
\end{tabular}

${ }^{a}$ Based on the redefined Minkowski subtraction.

${ }^{\mathrm{b}}$ Based on the original Minkowski subtraction.

the commonly agreed definition for an erosion is $\varepsilon$ but the same notation $\delta$ is used for both definitions of the dilation. Table 1 summarizes the existing definitions and notations currently used in mathematical morphology for erosions, dilations, and Minkowski operators.

Fortunately, everyone agrees on a common definition for the erosion. As concerns the dilation, several important practical reasons may be in favour of its first definition:

- As for the erosion, no reflection of the structuring element is required for defining the dilation.

- It is the dual transformation of the erosion with respect to set complementation: $\delta_{B}=\mathrm{C} \varepsilon_{B} \mathrm{C}$, where $\mathrm{C}$ denotes the complementation operator. When using the second definition for the dilation, one has to reflect the structuring element on one side of the equality: $\delta_{\breve{B}}=\mathrm{C} \varepsilon_{B} \mathrm{C}$ or $\delta_{B}=\mathrm{C} \varepsilon_{\breve{B}} \mathrm{C}$.

- It is a rank filter with the same structuring element (i.e., without having to reflect it), as is the erosion (see details in Section 2.3).

- It coincides with the usual definition of the so-called max filters [37] (as does the erosion with the min filters).

However, from a theoretical point of view, the concept of adjunction [49], recently introduced in mathematical morphology [50] for defining erosions and dilations operators in the general context of complete lattices [51], is in favour of the second definition of the dilation because it forms an adjunct pair with the commonly agreed definition for the erosion. Recall that two transformations $\psi_{1}$ and $\psi_{2}$ form an adjunction between the two complete lattices $\mathscr{L}$ and $\mathscr{M}$ if the following relationship holds:

$\psi_{1}(Y) \leqslant X \rightleftharpoons Y \leqslant \psi_{2}(X)$,

for every $X \in \mathscr{L}$ and $Y \in \mathscr{M}$. In this case, $\psi_{1}$ is called a dilation and $\psi_{2}$ an erosion [52]. Note that, with the adjunction relation, an erosion/dilation pair is defined without using the complementation (contrary to the duality relation with respect to set complementation recalled earlier). Nevertheless, the adjunction relation alone does not either uniquely define the erosion nor the dilation because it characterises a pair of transformations. For example, "Minkowski addition/Minkowski subtraction", "Serra's erosion by $B$ /Minkowski addition by $B$ ", and "Serra's dilation by $B /$ Minkowski subtraction by $\breve{B}$ " are all pairs of operators satisfying the adjunction relation.

In this paper, since we are dealing with relations between morphological and rank filters, it happens to be much more convenient to follow Serra's definition of the dilation. We also adopt functional notations. That is, the erosion $\varepsilon$ and dilation $\delta$ of a grey scale image $f$ by a flat structuring element $B$ positioned at a pixel $x$ are denoted and defined as follows:

$$
\begin{gathered}
{\left[\varepsilon_{B}(f)\right](x)=\min _{b \in B} f(x+b),} \\
{\left[\delta_{B}(f)\right](x)=\max _{b \in B} f(x+b) .}
\end{gathered}
$$

Erosion and dilations are nonlinear, increasing, and translation invariant filters. Erosions and dilations form dual pairs of transformations with respect to complementation. Erosions and dilations with flat structuring elements satisfy the threshold decomposition principle and commute with anamorphoses of the intensity values $\left[33\right.$, p. 436]. By construction, $\varepsilon_{B} \leqslant \delta_{B}$ but the anti-extensivity of the erosion and the extensivity of the dilation are verified if and only if the structuring element $B$ contains its origin:

$\varepsilon_{B} \leqslant \mathrm{id} \leqslant \delta_{B} \rightleftharpoons B$ contains its origin,

where id denotes the identity transformation. The dilation distributes the pointwise maximum operator and the erosion the pointwise minimum operators.

\subsection{Links}

Obviously, the erosion is equivalent to a rank filter of rank 1 and the dilation to a rank filter of rank $n$ where $n$ is 
the cardinal number of the structuring element (see details in Ref. [53, Section III]): $\varepsilon_{B}=\zeta_{B, 1}, \delta_{B}=\zeta_{B, n}$, where $n=\operatorname{card}(B)$. On the other hand, since rank filters are increasing and translation invariant, the representation theorem of Matheron [32] ${ }^{4}$ implies that any of them can be represented as a union of erosions (or, by duality with respect to set complementation, as an intersection of dilations). This led Maragos and Schafer [53] prove the following theorem (see Theorem 1 [53, p. 1172]): for any grey scale image $f$ and any finite structuring element $B$ containing $n$ points, the rank filter of order $k$ by $B$ is equal to the pointwise maximum of the erosions of $f$ by all $n ! /(k !(n-k) !)$ subsets of $B$ containing exactly $k$ points, or, by duality, it is equal to the pointwise minimum of the dilations of $f$ by all $n !(k !(n-k) !)$ subsets of $B$ containing exactly $n-k+1$ points.

Rank filters on binary images can be obtained by thresholding the convolution of the image with the chosen structuring element as convolution kernel [55-59] for all values larger or equal to $n-k+1$. This idea is also at the basis of the so-called $\Xi$-filters introduced by Preston [60] in 1983. More precisely, $\Xi$-filters use the threshold decomposition and stacking (i.e., increasing) properties $[18,40]$ of rank filters for processing grey scale images with a finite number of grey scale levels: the output image is obtained by summing up the thresholds of the convolution of each level set of the image. $\Xi$-filters may use an additional 'level parameter' which indicates whether a low rank/high rank or high rank/low rank sequence must be applied below or above the specified level. $\Xi$-filters are also defined for non-flat structuring element by thresholding the convolution of the image subgraph (called 'logical signal' in Ref. [60]) of the signal.

The regulated binary erosion and dilation operators recently introduced in Ref. [61] are simply rank order filters (although this link is already pointed in the latter paper, an actual equivalence with rank order filter would have appeared if Serra's definition of the dilation would have been adopted). The proposed strictness parameter can be directly related to the rank parameter of the corresponding rank filter. Likewise, the election operation with a voting threshold proposed in Ref. [62] for processing binary images is equivalent to a rank order filter with a rank equal to the voting threshold. Besides, it is shown in Ref. [62] that the generalization voting logic to grey scale images leads to rank order filters.

Rank filters have been generalized to weighted order statistic filters whereby weights are introduced in the window (structuring element). The weights are integer

\footnotetext{
${ }^{4}$ It is worth mentioning here that an even more general representation theorem whereby the increasing property is dropped has been proven later by Banon and Barrera [54]. This theorem states that any translation invariant mapping can be represented as a union of a family of hit-or-miss transformations.
}

values indicating how many times the corresponding value in the image must be duplicated before computing the chosen rank [40, Eq. (7)] (see Ref. [12, p. 188] for weighted median filters). Note that Wilson [62] proposed another definition of weighted rank order filters which is a generalization of erosions and dilations which non-flat structuring elements. More precisely, the reference point of the structuring element is placed at an image pixel; the output value being obtained by (1) adding the weights of the structuring element to the values of the corresponding image pixels, (2) ordering the resulting sums, and (3) selecting as output the $k$ th element in the sorted list.

Soft erosions and dilations introduced in Refs. [63-65] are a special case of weighted order statistic filters. Indeed, the basic idea of soft morphological operations is that the structuring element $B$ is split into two subsets: the core subset $A$ and the soft subset $B \backslash A$. Soft morphological erosion (dilation) of a grey scale image with respect to a finite discrete set $B$ at a pixel $x$ is defined by sorting in ascending (descending) order the $\operatorname{card}(B \backslash A)+k \operatorname{card}(A)$ values of the input image including the pixels inside $B \backslash A$ and repeating $k$ times the values of the pixels inside $A$, and then selecting the $k$ th order from the sorted list. Finally, beware that the binary soft erosions and dilations defined in Ref. [66] are in fact binary rank filters and not soft operators in the sense described before.

\section{Rank order based openings and closings}

Rank order filters are at the basis of very powerful openings and closings called rank-max openings and rank-min closings. These operations have been originally proposed by Ronse [67-69, p. 325] and later described in Ref. [70, pp. 193-196] and [35, pp. 201-202] (see also Ref. [71, p. 193] where they are called rank-openings and [36, pp. 98-99] where they are called parametric openings). Let us recall the definition of a rank-max opening, starting from its geometrical interpretation and then showing its equivalent formulation based on rank filters. Rather than using a plain discrete structuring element $B$ whose cardinal number equals $n$ pixels (i.e., $\operatorname{card}(B)=n$ ), the rank-max opening consists in unioning the morphological openings by all possible subsets $B_{i}$ of $B$ containing $k$ pixels $(1 \leqslant k \leqslant n)$. For discrete grey scale images, the union operator simply generalises to the pointwise maximum operator $\vee$. We denote by $\gamma_{B, k}$ the rank-max opening with a structuring element $B$ and parameter $k$ by $\gamma_{B, k}$ :

$\gamma_{B, k}=\bigvee_{i}\left\{\gamma_{B_{i}} \mid B_{i} \subseteq B\right.$ and $\left.\operatorname{card}\left(B_{i}\right)=k\right\}$,

where $1 \leqslant k \leqslant n$ and $n=\operatorname{card}(B)$. Notice that the smaller $k$ is, the less active is the corresponding rank-max 
opening. More precisely, the following ordering relationship holds: $\gamma_{B, n}=\gamma_{B} \leqslant \gamma_{B, n-1} \leqslant \cdots \leqslant \gamma_{B, 2} \leqslant \gamma_{B, 1}=\mathrm{id}$. From a computational point of view, it can be seen that the upper bound for the number of distinct structuring elements $B_{i}$ in Eq. (1) equals $n ! /(k !(n-k) !)$. This number is too large for most real applications. Fortunately, it can be shown (e.g., Ref. [35, pp. 201-202]) that the rank-max opening is equivalent to the intersection (pointwise minimum operator $\wedge$ ) between the identity transform and the dilation by $\breve{B}$ of the rank filter $\zeta$ using $B$ as kernel and $n-k+1$ as rank:

$\gamma_{B, k}=\mathrm{id} \wedge\left(\delta_{\breve{B}} \zeta_{B, n-k+1}\right)$

Rank-max openings deserve being called openings because they satisfy the three algebraic properties required for a morphological opening, i.e., increasingness, antiextensivity, and idempotence. This follows directly from their definition (Eq. (1)) because Matheron [32] has shown that any union of openings is itself an opening.

The practical interest of rank-max openings (and the dual rank-min closings) is illustrated in [67] for the processing of digitized X-ray angiographic images. Alternating sequential filters [44] based on rank-max openings and rank-min closings are investigated for filtering noisy binary images in Ref. [72]. Rank-max openings have also been used in Refs. [73,74] for the extraction of the laid and chain lines in paper watermarks, in Refs. [75,76] for the detection of the local orientation of thin elongated objects in noisy images using a bank of rank-max opening and rank-min closing filters, and in Ref. [77] for the filtering of vein networks appearing on the leaves of plants. All these applications deal with the direct processing of grey scale images. Fast algorithms for translation invariant rank-max openings by line segments in arbitrary directions are proposed in Ref. [76].

Note that Maragos [78] and Wilson [62] independently introduced the related concepts of rank opening ${ }^{5}$ [78] and shape inference transform [62]. These two transformations are in fact identical since they are both defined as a rank filter followed by a dilation with the (reflected) structuring element, i.e., the intersection between the identity transform and the rank opening (or, equivalently, the shape inference transform) leads to the rank-max opening. The term 'rank opening' [78] may be misleading in the sense that it is not an opening. Indeed, although it is an increasing transformation, it does not satisfies two additional properties required for an opening: the anti-extensivity and idempotence properties. In contrast, the so-called regulated opening for binary images proposed in Ref. [61, p. 962] is an opening. However, while it is very similar to a rank-max opening, it has

\footnotetext{
${ }^{5}$ Contrary to Serra [71, p. 193] who refers to actual rank-max openings using the term rank-opening.
}

inferior performances. Indeed, the regulated opening with a structuring element $B$ and strictness $s$ may be interpreted as the intersection between the input set and the union of all possible shifts of the structuring element $B$ for which the intersection with the shape is big enough and the origin of the kernel is included in the shape. The second condition is rather restrictive. Indeed, by doing so, the result of the regulated opening depends on the origin of the structuring element (remember that the output of an ordinary opening is independent of the position of the origin). In particular, the output of the regulated opening is the empty set if the origin is outside of the structuring element. In addition, the regulated opening of a set such by a structuring element such as a $2 \times 2$ square does not commute with rotations by a multiple of $\pi / 2$ of the input set because it is not possible to set the origin of a $2 \times 2$ square at its centre. In fact, by relaxing this second condition, i.e., by performing a regulated erosion (i.e., a rank filter) instead of an anti-extensive regulated erosion in the first step of the regulated opening [61, Eq. (74)], one obtains the already described rank-max opening.

Finally, note it is questionable whether soft openings (closings) $[64,65]$ should actually be called openings (closings) because although they do not satisfy all properties required for an opening (closing): although increasing, they are neither idempotent nor anti-extensive (extensive).

\section{Rank order based hit-or-miss transform}

The hit-or-miss transform [33] involves a structuring element composed of two sets: the first has to fit the object under study while the second has to miss it. In mathematical terms, the hit-or-miss transformation, $H M T$, of a set $X$ by a composite structuring element $B=\left(B_{1}, B_{2}\right)$ is the set of points, $x$, such that when the origin of $B$ coincides with $x, B_{1}$ fits $X$ and $B_{2}$ fits $X^{c}$ :

$H M T_{B}(X)=\left\{x \mid\left(B_{1}\right)_{x} \subseteq X,\left(B_{2}\right)_{x} \subseteq X^{c}\right\}$.

As suggested by Serra [33, p. 40] this definition can be immediately generalized to the case where $n \geqslant 2$ component sets $X_{i}$ are defined. Then every point $x$ has to fulfill as many conditions of the type $B_{i} \subseteq X_{i}$. Wilson [62] formalized in 1989 this idea while further generalizing it by allowing for partial inclusions. The resulting transform is called the vector rank order operator: any number $n$ of binary images $X_{i}$ and structuring elements $B_{i}$ can be considered, the output value at a given pixel being itself defined by sorting the output of each component processed in parallel by a rank filter with structuring element $B_{i}$ and rank $k_{i}$, and selecting a given rank $k$ :

$\zeta_{k}\left(\zeta_{B_{1}, k_{1}}\left(X_{1}\right), \ldots, \zeta_{B_{n}, k_{n}}\left(X_{n}\right)\right)$. 
A massively parallel system (SIMD) allowing for bit serial rank order filter algorithms is described in Refs. $[79,80]$. In Ref. [81], it is shown that a sequence of vector rank order operators (they are called rank-order hitand-miss operations in this later reference) are isomorphic to multilayer feed-forward translation invariant (iconic) neural networks with signals of 0 or 1 and weights \pm 1 . The theory of matrix morphology which encompasses vector operations such as the vector rank order filter is detailed in Ref. [82] and generalized later in Ref. [83].

Vector rank order operators for two image components, where $X_{1}=X$ and $X_{2}=X^{c}$ and by considering the intersection (i.e., minimum rank) for combining the images processed in parallel, have been rediscovered in 1990 in Ref. [84] (see Refs. [85,86] for an optical correlator realization) where they are called rank order hitor-miss and [87] where they are called rank hit-miss transform (see also Ref. [35, pp. 118-119]):

$\zeta_{B_{1}, k_{1}}(X) \cap \zeta_{B_{2}, k_{2}}\left(X^{c}\right)$

Similarly, the split threshold hit/miss transform proposed in Ref. [88] is nothing but a vector rank order operator whereby $X_{1}$ and $X_{2}$ are obtained by thresholding an input grey scale image for different grey level ranges:

$\zeta_{B_{1}, k_{1}}\left(X_{1}\right) \cap \zeta_{B_{2}, k_{2}}\left(X_{2}\right)$.

Finally, another similar transform called the regulated hit-or-miss transform has been defined recently in Ref. [61, p. 967]:

$\left(\zeta_{B_{1}, k_{1}}(X) \cap X\right) \cap \zeta_{B_{2}, k_{2}}\left(X^{c}\right)$.

Therefore, regulated hit-or-miss and rank hit-miss (or the more general vector rank order) filters only differ by the fact that an anti-extensive regulated erosion is considered in the left term of Eq. (6) instead of a regulated erosion (remember that both regulated erosions and dilations come down to a rank filter, see Section 2.3).

Ranked hit-or-miss like transforms are best suited for recognition tasks where the shape of the searched objects are approximatively known and/or may be corrupted by external factors. First applications dealt with optical character recognition tasks $[62,81]$. Complete systems for automatic target recognition are described in Ref. [84,88].

\section{Concluding remarks}

We have reviewed all morphological operators based on rank order filters. Links and sometimes equivalences

Table 2

Rank order based morphological operators: synoptic table

\begin{tabular}{ll}
\hline Transformation & Definition and/or comment \\
\hline 2-D $\Xi$-filter ${ }^{\mathrm{a}}[60]$ & Equivalent to rank filter \\
3-D $\Xi$-filter ${ }^{\mathrm{a}}[60]$ & $\begin{array}{l}\text { Thresholded convolution on subgraph (equivalent to binary rank filter } \\
\text { on image subgraph) }\end{array}$ \\
Voting logic [62] & Equivalent to binary rank filter \\
Generalized voting logic [62] & Equivalent to rank filter \\
Regulated erosion/dilation [61] & Equivalent to binary rank filter \\
Soft erosion/dilation [63] & Special case of weighted order statistic filter (weight $k$ for the hard part \\
& and weight 1 for the soft part of the composite struct. elem.) \\
Weighted rank order filter [62] & Generalization of morphology with non-flat struct. elem. \\
& $($ different from weighted order statistic filters!) \\
Rank-max opening [67] (also called & $\bigvee_{i}\left\{\gamma_{B_{i}} \mid B_{i} \subseteq B\right.$ and card $\left.\left(B_{i}\right)=k\right\}$ \\
rank-opening in Ref. [71] and parametric & $=$ id $\wedge\left(\delta_{\breve{B}} \zeta_{B, n-k+1}\right)$ (it is an opening) \\
opening in Ref. [36]) & \\
Rank opening [78] & $\delta_{\breve{B}} \zeta_{B, n-k+1}($ it is not an opening!) \\
Shape inference transform [62] & $\delta_{\breve{B}} \zeta_{B, n-k+1}$ \\
Soft opening [63] & In fact, it is not an opening (see text) \\
Vector rank order operator [62] & $\zeta_{k}\left(\zeta_{B_{1}, k_{1}}\left(X_{1}\right), \ldots, \zeta_{B_{n}, k_{n}}\left(X_{n}\right)\right)$ \\
Rank order hit-or-miss [84] & $\zeta_{B_{1}, k_{1}}(X) \cap \zeta_{B_{2}, k_{2}}\left(X^{c}\right)$ \\
Rank hit-miss [87] & $\zeta_{B_{1}, k_{1}}(X) \cap \zeta_{B_{2}, k_{2}}\left(X^{c}\right)$ \\
Split threshold hit/miss [88] & $\zeta_{B_{1}, k_{1}}\left(X_{1}\right) \cap \zeta_{B_{2}, k_{2}}\left(X_{2}\right)$, where $X X_{1}$ and $X_{2}$ are obtained by performing \\
& two thresholds on an input grey scale image \\
Regulated hit-or-miss [61] & $\left(\zeta_{B_{1}, k_{1}}(X) \cap X\right) \cap \zeta_{B_{2}, k_{2}}\left(X^{c}\right)$ \\
\hline &
\end{tabular}

${ }^{a}$ Without considering the level parameter. 
between these operators have been highlighted. Most of them are summarized in Table 2. Rank based morphological operators are important for real applications because they are often more robust to noise and shape variations than morphological operators with plain structuring elements. We hope that this short compendium will foster further research and contribute to the dissemination of these useful, yet little known (as illustrated by their recurrent rediscovery in the literature), operators.

Eventually, it seems that common names for common transformations would certainly ease the development of a coherent theory. For example, we could perhaps adopt the following taxonomy 'rank opening' (for the identical transformations known as the rank-max, parametric, and rank opening), 'rank closings' (for the dual transformation), 'rank hit-or-miss' for the basic generalization (Eq. (5)) of the hit-or-miss transform and "vector rank hit-or-miss' for the more general transform (Eq. (4)).

\section{Acknowledgements}

The author wishes to thank the referees for their very stimulating comments and suggestions and Henk Heijmans for his advice to include a discussion about the concept of adjunction.

\section{References}

[1] A. Sarhan, B. Greenberg (Eds.), Contributions to Order Statistics, Wiley, New York, 1962.

[2] H. David, Order statistics, Wiley, New York, 1970.

[3] J. Tukey, Nonlinear (nonsuperposable) methods for smoothing data, In Cong. Rec., EASCON, 1974, p. 673.

[4] J. Tukey, Exploratory Data Analysis, Addison-Wesley, Reading, MA, 1977.

[5] J. Fitch, E. Coyle, N. Gallagher, The analog median filter, Trans. Circuits Systems 33 (1) (1986) 94-102.

[6] H. Longbotham, A. Bovik, Comments on the analog median filter, Trans. Circuits Systems 36 (1989) 310.

[7] S. Paul, K. Huper, Analog rank filtering, IEEE Trans. Circuits Systems I-Fund. Theory Appl. 40 (7) (1993) 469-476.

[8] G. Wecksung, K. Campbell, Digital image processing at EG\&G, Computer 7 (1974) 63-71.

[9] B. Frieden, A new restoring algorithm for the preferential enhancement of edge gradients, J. Opt. Soc. Amer. 66 (3) (1976) 280-283.

[10] L. Rabiner, M. Sambur, C. Schmidt, Applications of nonlinear smoothing algorithm to speech processing, Trans. Acoust. Speech Signal Process. 23 (6) (1975) 552-557.

[11] N. Jayant, Average and median-based smoothing techniques for improving digital speech quality in the presence of transmission errors, IEEE Trans. Commun. 24 (1976) 1043-1045.

[12] B. Justusson, Median filtering: statistical properties, in: T. Huang (Ed.), Two-Dimensional Digital Signal Processing
II: Transforms and Median Filters, Topics in Applied Physics. Vol. 43, Springer, Berlin, 1981, pp. 161-196 (chapter 5).

[13] R. Duin, W. Haringa, R. Zeelen, Fast percentile filtering, Pattern Recognition Lett. 4 (1986) 269-272.

[14] G. Heygster, Rank filters in digital image processing, Comput. Graph. Image Process. 19 (2) (1982) 148-164.

[15] H. Tagare, R. de Figueiredo, Order Filters, Proc. IEEE 73 (1985) 163-165.

[16] A. Bovik, T. Huang, D. Munson Jr., A generalization of median filtering using linear combinations of order statistics, IEEE Trans. Acoust. Speech, Signal Process. 31 (6) (1983) 1342-1350.

[17] T. Nodes, N. Gallagher, Median filters: some modifications and their properties, IEEE Trans. Acoust. Speech Signal Process. 30 (1982) 739-746.

[18] J. Fitch, E. Coyle, N. Gallagher, Threshold decomposition of multidimensional ranked order operations, IEEE Trans. Circuits Systems 32 (1985) 445-450.

[19] P. Salembier, Adaptive rank order based filters, Signal Process. 27 (1) (1992) 1-25.

[20] G. Heygster, Untersuchung von zweidimensionalen Rangordnungsoperatoren im Orts - und Frequenzbereich, in: E. Triendl (Ed.), Bildverarbeitung and Mustererkennung, Informatik Fachberichte, Vol. 17, Springer, Berlin, Heidelberg, 1978, pp. 204-208.

[21] R. Hodgson, D. Bailey, A. Naylor, S. McNeill, Properties, implementations and applications of rank filters, Image Vis. Comput. 3 (1) (1985) 4-14.

[22] I. Pitas, A. Venetsanopoulos, Order statistics in digital signal processing, Proc. IEEE 80 (12) (1992) 1893-1921.

[23] M. Gabbouj, E. Coyle, N. Gallagher, An overview of median and stack filtering, Circuits Systems Signal Process. 11 (1) (1992) 7-45.

[24] A. Fisher, Systolic algorithms for running order statistics in signal and image processing, J. Dig. Systems 6 (1982) 251-264.

[25] L. Lucke, K. Parhi, Parallel processing architectures for rank order and stack filters, IEEE Trans. Signal Process. 42 (5) (1994) 1178-1189.

[26] A. Gasteratos, I. Andreadis, P. Tsalides, Realization of rank order filters based on majority gate, Pattern Recognition 30 (9) (1997) 1571-1576.

[27] A. Gasteratos, I. Andreadis, Non-linear image processing in hardware, Pattern Recognition 33 (6) (2000) 1013-1021.

[28] G. Garibotto, L. Lambarelli, Fast on-line implementation of two dimensional median filtering, Electron. Lett. 15 (1) (1979) 24-25.

[29] T. Huang, G. Yang, G. Tang, A fast two-dimensional median filtering algorithm, IEEE Trans. Acoust. Speech Signal Process. 27 (1) (1979) 13-18.

[30] B. Chaudhuri, An efficient algorithm for running window pel gray level ranking in 2D images, Pattern Recognition Lett. 11 (2) (1990) 77-80.

[31] M. Van Droogenbroeck, H. Talbot, Fast computation of morphological operations with arbitrary structuring elements, Pattern Recognition Lett. 17 (14) (1996) 1451-1460.

[32] G. Matheron, Random Sets and Integral Geometry, Wiley, New York, 1975.

[33] J. Serra, Image Analysis and Mathematical Morphology, Academic Press, London, 1982. 
[34] J. Serra (Ed.), Image analysis and mathematical morphology. vol. 2. Theoretical Advances, Academic Press, London, 1988.

[35] H. Heijmans, Morphological image operators, Advances in Electronics and Electron Physics, Academic Press, Boston, 1994.

[36] P. Soille, Morphological Image Analysis, Springer, Berlin, 1999.

[37] Y. Nagakawa, A. Rosenfeld, A note on the use of local min and max operations in digital picture processing, IEEE Trans. Systems Man Cybernet. 8 (1978) 632-635.

[38] V. Goetcherian, From binary to grey tone image processing using fuzzy logic concepts, Pattern Recognition 12 (1980) 7-15.

[39] I. Bloch, H. Maître, Fuzzy mathematical morphologies: a comparative study, Pattern Recognition 28 (9) (1995) 1341-1387.

[40] P. Wendt, E. Coyle, N. Gallagher, Stack filters, IEEE Trans. Acoust. Speech Signal Process. 34 (4) (1986) 898-911.

[41] P. Salembier, Size-sensitive multiresolution decomposition of images with rank order based filters, Signal Process. 27 (1992) 205-241.

[42] P. Maragos, R. Schafer, Morphological systems for multidimensional signal processing, Proc. IEEE 78 (4) (1990) 690-709.

[43] G. Matheron, Eléments pour une Théorie des Milieux Poreux, Masson, Paris, 1967.

[44] S. Sternberg, Grayscale morphology, Comput. Graph. Image Process. 35 (1986) 333-355.

[45] R. Haralick, S. Sternberg, X. Zhuang, Image analysis using mathematical morphology, IEEE Trans. Pattern Anal. Mach. Intell. 9 (4) (1987) 532-550.

[46] A. Rosenfeld, A. Kak, Digital Picture Processing, vol. 2, 2nd Edition, Academic Press, Orlando, 1982.

[47] H. Hadwiger, Minkowskische Addition und Subtraktion beliebiger Punktmengen und die Theoreme von Erhard Schmidt, Math. Z. 53 (3) (1950) 210-218.

[48] H. Minkowski, Über die begriffe Länge, Oberfläche und Volumen, Jahresh. Dsch. Math. Ver. 9 (1901) 115-121.

[49] G. Gierz, K. Hofmann, K. Keimel, J. Lawson, M. Mislove, D. Scott, A Compendium of Continuous Lattices, Springer, Berlin, 1980.

[50] H. Heijmans, C. Ronse, The algebraic basis of mathematical morphology: I. Dilations and erosions, Comput. Vis. Graph. Image Process. 50 (1990) 245-295.

[51] G. Birkhoff, Lattice Theory, vol. 25, American Mathematical Society Colloquium Publications, American Mathematical Society, Providence, RI, 1967.

[52] H. Heijmans, Mathematical morphology: a modern approach in image processing based on algebra and geometry, SIAM Rev. 37 (1) (1995) 1-36.

[53] P. Maragos, R. Schafer, Morphological filters-Part II: Their relations to median, order-statistic, and stack filters, IEEE Trans. Acoust. Speech Signal Process. 35 (8) (1987) $1170-1184$.

[54] G. Banon, J. Barrera, Minimal representation for translation-invariant set mappings by mathematical morphology, SIAM J. Appl. Math. 51 (1991) 1782-1798.

[55] K. Preston Jr., Multidimensional logical transforms, IEEE Trans. Pattern Anal. Machine Intell. 5 (5) (1983) 539-554.
[56] F. Gerritsen, P. Verbeek, Implementation of cellular-logic operators using a $3 \times 3$ convolution and table lookup hardware, Comput. Vis. Graph. Image Process. 27 (1984) 115-123.

[57] J. Mazille, Mathematical morphology and convolutions, J. Microsc. 156 (Pt1) (1989) 3-13.

[58] F. Shih, O. Mitchell, Threshold decomposition of grayscale morphology into binary morphology, IEEE Trans. Pattern Anal. Machine Intell. 11 (1) (1989) 31-42.

[59] M. Killinger, J.-L. de Bougrenet, P. Cambon, C. Le Moing, Morphological filtering using a Fourier transform hologram, Opt. Commun. 73 (6) (1989) 434-438.

[60] K. Preston Jr., $\Xi$-Filters, IEEE Trans. Acoust. Speech Signal Process. 31 (4) (1983) 861-876.

[61] G. Agam, I. Dinstein, Regulated morphological operations, Pattern Recognition 32 (6) (1999) 947-971.

[62] S. Wilson, Vector morphology and iconic neural networks, IEEE Trans. Systems Man Cybernet. 19 (6) (1989) 1636-1644.

[63] L. Koskinen, J. Astola, Y. Neuvo, Soft morphological filters, In P. Gader, E. Dougherty (Eds.), Image Algebra and Morphological Image Processing II, vol. SPIE-1568, (1991) pp. 262-270.

[64] L. Koskinen, J. Astola, Soft morphological filters: a robust morphological filtering method, J. Electron. Imag. 3 (1) (1994) 60-70.

[65] P. Kuosmanen, J. Astola, Soft morphological filtering, J. Math. Imag. Vis. 5 (3) (1995) 231-262.

[66] M. Zmuda, L. Tamburino, Efficient algorithms for the soft morphological operators, IEEE Trans. Pattern Anal. Machine Intell. 18 (11) (1996) 1142-1147.

[67] C. Ronse, Erosion of narrow image features by combination of local low rank and max filters, Proceedings of Second IEE International Conference on Image Processing and its Applications, London, 1986, pp. 77-81.

[68] C. Ronse, Extraction of narrow peaks and ridges in images by combination of local low rank and max filters: implementation and applications to clinical angiography. Working Document WD47, Philips Research Laboratory, Bruxelles, 1988. (Reprints may be asked for by writing an email to the author ronse@dpt-info.ustrasbg.fr)

[69] C. Ronse, Order-configuration functions: Mathematical characterizations and applications to digital signal and image processing, Inform. Sci. 50 (3) (1990) 275-327.

[70] C. Ronse, H. Heijmans, The algebraic basis of mathematical morphology: II. Openings and closings, Comput. Vis. Graph. Image Process. Image Understanding 54 (1) (1991) 74-97.

[71] J. Serra, Dilation and filtering for numerical functions, in: J. Serra (Ed.), Image Analysis and Mathematical Morphology. vol. 2: Theoretical Advances. Academic Press, New York, 1988, pp. 181-202, (chapter 9).

[72] H. Heijmans, Morphological filters for dummies, in: P. Maragos, R. Schafer, M. Butt (Eds.), Mathematical Morphology and its Applications to Image and Signal Processing, Kluwer Academic Publishers, Dordrecht, 1996, pp. 127-137.

[73] P. Whelan, P. Soille, Watermark extraction in paper samples, in: D. Vernon (Ed.), Proceedings of Optical Engineering Society of Ireland and Irish Machine Vision and Image 
Processing Joint Conference, National University of Ireland, Maynooth, September 1998, pp. 287-299.

[74] P. Whelan, P. Soille, A. Drimbarean, Real-time registration of paper watermarks, Real-Time Imaging 7 (4) (2001).

[75] P. Soille, H. Talbot, Image structure orientation using mathematical morphology, in: A. Jain, S. Venkatesh, B. Lovell (Eds.), 14th International Conference on Pattern Recognition, vol. 2, IAPR, IEEE Computer Society, Brisbane, August, 1998, pp. 1467-1469.

[76] P. Soille, H. Talbot, Directional morphological filtering, IEEE Trans. Pattern Anal. Machine Intelli., submitted for publication.

[77] P. Soille, Morphological image analysis applied to crop field mapping, Image and Vision Computing, 18(13):1025-1032, October 2000

[78] P. Maragos, Optimal morphological approaches to image matching and object detection, in: Second International Conference on Computer Vision, Tampa, FL, December 5-8, 1988, Computer Society Press, Washington, DC, 1988, pp. 695-699.

[79] L. Schmitt, S. Wilson, The AIS-5000 parallel processor, IEEE Trans. Pattern Anal. Machine Intell. 10 (3) (1988) 320-330.

[80] S. Wilson, One dimensional SIMD architectures-The AIS-5000, in: S. Levialdi (Ed.), Multicomputer Vision, Proceedings of the Eighth Workshop on Multicomputers, Rome, Italy, June 2-5, 1987, Academic Press, London, 1988, pp. 131-149.
[81] S. Wilson, Training structuring elements in morphological networks, in: E. Dougherty (Ed.), Mathematical Morphology in Image Processing, vol. 34, Optical Engineering, Marcel Dekker, New York, 1993, pp. 1-41 (chapter 1).

[82] S. Wilson, Theory of matrix morphology, IEEE Trans. Pattern Anal. Machine Intell. 14 (1992) 636-652.

[83] J. Goutsias, H. Heijmans, K. Sivakumar, Morphological operators for image sequences, Comput. Vis. Image Understand. 62 (1995) 326-346.

[84] D. Casasent, R. Sturgill, Optical hit-or-miss morphological transforms for ATR, in: A. Tescher (Ed.), Applications of Digital Image Processing XII, vol. SPIE-1153, 1990, pp. $500-510$.

[85] D. Casasent, R. Schaefer, R. Sturgill, Optical hit-miss morphological transform, Appl. Opt. 31 (29) (1992) 6255-6263.

[86] D. Casasent, General-purpose optical pattern recognition image processors, Proc. IEEE 82 (11) (1994) 1724-1734.

[87] D. Bloomberg, P. Maragos, Generalized hit-miss operations, in: P. Gader (Ed.), Image Algebra and Morphological Image Processing, vol. SPIE-1350, 1990, pp. 116-128.

[88] J. Gilmour, D. Casasent, Split threshold hit/miss transform for mines, in: A. Dubey, C. Barnard, C. Lowe, J. McFee (Eds.), Proceedings of the SPIE Conference on Detection Technologies for Mines and Minelike Targets, vol. 2765, Orlando, FL; 1996, SPIE, pp. 767-787.

\begin{abstract}
About the Author-PIERRE SOILLE received the engineering degree from the Universite de Louvain in 1988. Research on the processing of digital elevation models and satellite images provided the material for the doctoral degree he gained in 1992 at the same university and in collaboration with the Centre de Morphologie Mathématique of the Ecole des Mines de Paris. He then pursued research on image analysis and mathematical morphology at the CSIRO Mathematical and Information Sciences Division, Sydney, the Centre de Morphologie Mathématique, Fontainebleau, and the Abteilung Mustererkennung of the Fraunhofer Institut IPK, Berlin. In 1994, he co-organised with Jean Serra the Second International Symposium on Mathematical Morphology. During the period 1995-1998, he has been a lecturer and research scientist at the Laboratoire de Génie Informatique et d'Ingénierie de la Production of the Ecole des Mines d'Alès and EERIE, Nîmes. In 1999, he has been senior research scientist at the Silsoe Research Institute, UK, leading a project on the application of novel image analysis techniques to agri-food industries. Pierre is now with the EC Joint Research Centre, Space Applications Institute, Italy.

His contributions to the analysis of images through mathematical morphology have been at the basis of the 'Habilitation à Diriger des Recherches' degree he received in 1997 from the Université Montpellier II. He worked on many applied projects and contributed to research publications on image segmentation, interpolation, pattern recognition, shape analysis, efficient algorithms, and scientific/industrial applications. He has taught tutorials on mathematical morphology in conjunction with several international conferences. Pierre is author of the book Morphological Image Analysis: Principles and Applications recently published in English and German. [URL: http://ams.egeo.sai.jrc.it/soille].
\end{abstract}

